\section{Effects of statin use on functional capacity and muscle mass in elderly women}

\author{
Martine Boetje,1,2 Daniel Bunout,1 \\ Gladys Barrera,1 Maria Pia de la Maza,1 \\ Laura Leiva,1 Sandra Hirsch1 \\ 1Institute of Nutrition and Food \\ Technology (INTA), University of Chile, \\ Santiago, Chile; 2Wageningen University, \\ Division of Human Nutrition, \\ Wageningen, the Netherlands
}

\section{Abstract}

3-Hydroxy-3-methylglutaryl-coenzyme A (HMG-CoA) reductase inhibitors (statins) may produce mild to severe myopathy. However, it is unclear if sarcopenia (decreased muscle mass and functional capacity) is also accentuated by the drugs. In a cross-sectional design, functional capacity (timed up and go, quadriceps- and hand grip strength) and lean body mass were compared between 71 low-dose statin users $(<20 \mathrm{mg} /$ day $)$ and 57 age- and BMI-matched control subjects (all apparently healthy elderly women aged $74.6 \pm 5.3$ years). Possible confounding factors were assessed by questionnaires. A fasted blood sample was taken to measure serum creatine kinase levels to search for muscle damage. Statin users had higher functional capacity than controls (quadriceps strength $25.3 \pm 7.5 \mathrm{~kg}$ vs. $20.5 \pm 6.3$ $\mathrm{kg}$ in controls, $\mathrm{P}<0.001$; timed up and go $9.7 \pm 2.1 \mathrm{~s}$ vs. $10.8 \pm 2.6 \mathrm{~s}$ in controls, $\mathrm{P}=0.01$ ). After adjusting for confounding factors, this effect was not created by statin intake but by a difference in socioeconomic status between the two groups. Hand grip strength $(20.6 \pm 4.3$ $\mathrm{kg}$ vs. $19.9 \pm 3.8 \mathrm{~kg}, \mathrm{P}=0.47$ ) and lean body mass $(36.3 \pm 4.1 \mathrm{~kg}$ vs. $35.6 \pm 4.6 \mathrm{~kg}, \mathrm{P}=0.19)$ did not differ between statin users and controls. Muscle mass and functional capacity were not affected by statin intake but by socioeconomic status in elderly women.

\section{Introduction}

Statins are one of the most widely used drugs worldwide to treat or prevent cardiovascular disease. In the United States of America, $35.9 \%$ of people with high levels of cholesterol used statins in 2003-2004, ${ }^{1}$ which corresponds to $>100$ million prescriptions of statins. Since the prevalence of cardiovascular disease is rising and the elderly population is growing, the use of statins will continue to rise. The principal mechanism of cholesterol lowering of these drugs is via inhibition 3-hydroxy-3-methylglutaryl coenzyme A (HMG-CoA) reductase. Besides cholesterol lowering effects, statins also improve endothelial conditions and reduce systemic inflammation. These multiple effects explain the popularity of these drugs.

Despite its benefits, adverse effects like myopathy (muscle pain or weakness, with or without creatine kinase (CK) elevation) have been reported in $10 \%$ of high dose $(>40 \mathrm{mg}$ ) statin users. ${ }^{2}$ Statins may initiate immunemediated forms of necrotizing and inflammatory myopathy, ${ }^{3}$ thereby unmasking or aggravating various metabolic myopathies and other neuromuscular disorders. Rhabdomyolysis, the most severe form of myopathy, is reported in $0.5 \%$ of high dose statin users. ${ }^{4}$ Symptoms of myopathy get worse during exercise: muscle pain (myalgia), cramps, fatigue and weakness are then reported in $25 \%$ of statin users. ${ }^{5}$ Mitochondrial dysfunction might be the underlying cause of these adverse effects. ${ }^{6}$ Other side effects of statin use include headaches, nausea, abdominal pain and diarrhea. ${ }^{7}$

Sarcopenia is defined as the loss of muscle mass and function that occurs during normal aging. It is associated with a deterioration of muscle strength and walking capacity leading to dependence and increased mortality in older adults. ${ }^{8}$ Given that both statin use and sarcopenia are common with increasing age, the aim of this study was to investigate if statins also contribute to deterioration of functional capacity and body muscle mass in elderly subjects, besides subjective side effects like muscle pain.

\section{Materials and Methods}

\section{Study design}

A cross-sectional study was performed using 2 groups: elderly women currently taking statin treatment and a control group with similar age and body mass index (BMI).

\section{Study population}

The study population consisted of 128 community living elderly women, aged 65 years or more. One group consisted of 71 subjects currently taking low-dose ( $\leq 20 \mathrm{mg}$ ) statin treatment (for at least 6 months before measurements). The second group consisted of 57 control subjects without current statin intake (or with statin intake until $>2$ months before measurements). These time frames of statin use were chosen, because the majority of statin-related myopathy cases occur within the first six months of treatment and decreases within two months after cessation of the therapy. ${ }^{9}$ Subjects were drawn from the INTA database containing apparently healthy elderly
Correspondence: Sandra Hirsch, Institute of Nutrition and Food Technology (INTA), University of Chile, PO Box 138-11, Santiago, Chile.

Tel: +56.29781485 - Fax: +56.22214030 .

E-mail: shirsch@inta.cl

Key words: statin, myopathy, functional capacity, muscle mass, socioeconomic status.

Received for publication: 30 March 2011

Revision received: 29 August 2011.

Accepted for publication: 9 September 2011.

This work is licensed under a Creative Commons Attribution NonCommercial 3.0 License (CC BYNC 3.0).

(C) Copyright M. Boetj et al., 2011

Licensee PAGEPress, Italy

Ageing Research 2011; 3:e7

doi:10.4081/ar.2011.e7

subjects living in 2 different communities of Santiago (Chile): Macul (low socioeconomic status) and Las Condes (high socioeconomic status). Participants from Macul were collected from a public health centre. Participants from Las Condes were collected from three governmental elderly centers. Exclusion criteria were: severe movement disabilities, dementia (mini mental state $<20$ ), 10 taking medications that could interfere with the effects of statins on muscle (fibrates, corticoids) or presence of severe chronic diseases (cancer, AIDS, heart-, liver-, kidney- or lung failure). A minimal sample size of 60 subjects per group was calculated sufficient to yield a significant difference in functional capacity (20\% difference in TUG and 10\% in quadriceps strength) between the two groups (using a one sided $\alpha<0.05$ and a power of $80 \%$ ). The numbers were based on a previous INTA study measuring quadriceps strength and timed up and go in a general population of apparently healthy elderly in Santiago, Chile.11

\section{Study protocol}

Subjects came once to the premises (INTA) after an overnight fast. First, they had to sign a written informed consent after oral and written explanation of the study. Second, a fasted blood sample was taken in which creatine kinase was assessed. Third, after breakfast, mini nutritional assessment 12 and mini mental state ${ }^{10}$ questionnaires were performed, as well as a short questionnaire including questions about physical activity, diseases, medication use, muscle pain and nutrition. The purpose of these questionnaires was to identify possible confounders that could influence the outcome of the study, such as physical activity or muscle pain. Muscle pain was defined as chronic ( $>2$ weeks) pain in arm or leg mus- 
cles. Fourth, body examinations were performed including anthropometric measures and whole body densitometry. Finally, functional capacity was measured using a quadriceps table, a handgrip dynamometer and the timed up and go test. The study was approved by INTA's ethical committee.

\section{Methods}

\section{Blood sample}

A fasted blood sample of $10 \mathrm{~mL}$ was drawn from the ante-cubital vein in the forearm and stored in 2 tubes of $3 \mathrm{~mL}$ of serum and one tube $(4 \mathrm{~mL})$ for plasma. One tube of $3 \mathrm{~mL}$ serum was immediately stored in a dark environment) for creatine kinase measurement. One tube of serum and plasma were stored as reserve. Total creatine kinase (CK) was measured using automated techniques in a clinical laboratory (Vida Integra) and expressed in serum in International units per liter (IU/L). Creatine kinase is an indicator of muscle damage (3-5 x normal upper limit in myalgia, $>10 \mathrm{x}$ normal upper limit in rhabdomyolysis).

\section{Body composition and anthropometry}

Lean body mass (total and in legs and arms) was measured by double beam X-ray absorptiometry (DEXA) in Lunar Prodigy Equipment (General Electric Medical Systems, Madison, WI, USA). Afterwards, the following anthropometric measures were assessed: height $(\mathrm{cm})$, weight $(\mathrm{kg})$, body mass index $\left(\mathrm{kg} / \mathrm{m}^{2}\right)$ and knee-ankle distance $(\mathrm{cm})$.

\section{Functional capacity}

Functional capacity was assessed by measuring quadriceps isokinetic strength, hand grip isometric strength and the timed up and go (TUG) test. Quadriceps strength (lower extremity strength) was measured three times using a quadriceps table and a digital force transducer, as previously described. ${ }^{13}$ This method is preferable over the quadriceps hand-held dynamometer, since there is no need of force from the examiner. ${ }^{14}$ Maximal voluntary concentric isokinetic torque was measured at an angle of $30^{\circ}$, to maximize force and minimize muscle and knee pain 15 after a warming up session of 20 (suboptimal) muscle contractions. Subjects had to sit straight, lean with their back on the quadriceps table with their arms crossed in front of their chest, to prevent contribution of other muscle groups. A pause of 2 min was held between each measurement.

Quadriceps muscle torque was calculated using the formula:

Quadriceps muscle torque (Newton) $=\left(\mathrm{A}^{*} \mathrm{~B}\right) / \mathrm{C}$ $\mathrm{A}=$ Strength measured by digital force transducer $(\mathrm{N})$

$\mathrm{B}=$ Height quadriceps table $(\mathrm{cm})$

$\mathrm{C}=$ Distance knee-ankle $(\mathrm{cm})$
Quadriceps muscle torque was converted from Newtons into kilograms dividing by 9.8 (gravity velocity in $\mathrm{m} / \mathrm{s}^{2}$ ). Since leg muscle mass influences leg muscle force, quadriceps muscle torque was divided by kilograms leg lean body mass and expressed as quadriceps specific force $(\mathrm{kg} / \mathrm{kg})$. An inter-examiner reliability study showed no significant differences between examiners (variability 5\%) and a within-participant coefficient of variation, which ranged around $8 \%$, when measured in five subjects on five subsequent days (data not shown). Handgrip strength was measured using a handgrip dynamometer (Therapeutic Instruments, Clifton, NJ, USA). Maximal strength was expressed in kilograms and measured three times in each hand. Handgrip strength was corrected for arm lean body mass $(\mathrm{kg})$ and expressed as hand specific force $(\mathrm{kg} / \mathrm{kg}$ ). Timed up and go (TUG) (the time a subject can stand up from a chair, and walk around a piece of tape 4 meters away (total distance 8 meters)) was also measured, and expressed in seconds (better performance the lower the time required for the test). ${ }^{16}$

\section{Other covariates}

Confounders were identified using a specially developed questionnaire for the study and included questions about drug- and supplement intake, physical activity, muscle pain and co morbid conditions. Subjects were considered physically active if they performed physical activities more than two times per week.

\section{Statistical analysis}

Statistical analyses were performed using Stata 9.1 for Windows (Stata Corporation, Texas, USA). Normality of the variables was checked using normal probability plots and skewness/kurtosis normality tests. Variables with a normal distribution were expressed as mean \pm standard deviation. Differences in means of functional capacity and total body muscle mass between the two groups (statin users vs. controls) were assessed using a twosided independent student t-test in case of a normal distribution of the parameters. A Chisquare test was used for binominal categorical variables (physically active, high socioeconomic status, muscle pain). Kruskal-Wallis was used to test for non-parametric variables (age, BMI, fat mass, maximal hand grip strength, timed up and go, lean body mass). Correlations were assessed using Spearman rank. Multiple linear regression was used to examine the relationship of statin intake and functional capacity, adjusting for age, BMI, physical activity, muscle pain and socioeconomic status.

A P-value of $<0.05$ was considered statistically significant and marked in italic.

\section{Results}

\section{Subject characteristics}

A total of 137 subjects were eligible for the study and signed the written informed consent. Nine subjects were excluded, two because of a mini mental score $<20$, three because of a significant functional limitation and four because of an age $<65$ years. Finally, 128 subjects were included for statistical analyses (71 statin users and 57 controls). Among the former, 59 used atorvastatin, 8 lovastatin, 2 simvastatin and 2 rosuvastatin. Duration of statin use ranged from seven months to 20 years. Among the control subjects, three used statins until 2 months before measurements. Statin users and control subjects were similar in age (ranging from 65-84

Table 1. Basal descriptive characteristics of studied subjects.*

\begin{tabular}{lccc} 
Variable & Statin users $(\mathrm{N}=71)$ & Controls $(\mathrm{N}=57)$ & $\mathrm{P}$ \\
Age (years) & $74.3 \pm 5.2$ & $74.9 \pm 5.4$ & 0.54 \\
BMI $\left(\mathrm{kg} / \mathrm{m}^{2}\right)$ & $26.3 \pm 4.2$ & $27.5 \pm 5.7$ & 0.29 \\
\hline Mini Nutritional Assessment (0-29) & $26.1 \pm 2.3$ & $25.5 \pm 2.6$ & 0.46 \\
Physically active (n) & 54 & 31 & 0.01 \\
\hline High socioeconomic status (n) & 49 & 23 & 0.001 \\
\hline
\end{tabular}

*Values expressed as mean \pm standard deviation. Significant P-values $(<0.05)$ are presented in italic

Table 2. Functional capacity comparing statin users and control subjects.*

\begin{tabular}{lcrc}
\hline Variable & Statin users $(\mathrm{N}=7 \mathbf{1})$ & Controls $(\mathrm{N}=57)$ & $\mathrm{P}$ \\
Maximal quadriceps torque $(\mathrm{kg})$ & $25.3 \pm 7.5$ & $20.5 \pm 6.3$ & $<0.001$ \\
Quadriceps specific force $(\mathrm{kg} / \mathrm{kg})$ & $2.3 \pm 0.7$ & $1.9 \pm 0.6$ & $<0.001$ \\
\hline Maximal hand grip strength $(\mathrm{kg})$ & $20.6 \pm 4.3$ & $19.9 \pm 3.8$ & 0.47 \\
Hand specific force $(\mathrm{kg} / \mathrm{kg})$ & $5.8 \pm 1.2$ & $5.8 \pm 1.1$ & 0.80 \\
\hline Timed up and go $(\mathrm{sec})$ & $9.7 \pm 2.1$ & $10.8 \pm 2.6$ & 0.01 \\
Total lean body mass $(\mathrm{kg})$ & $36.3 \pm 4.1$ & $35.6 \pm 4.6$ & 0.19 \\
\hline *Values expressed as mean \pm standard deviation. Significant P-values $(<0.05)$ are presented in italic. & &
\end{tabular}

*Values expressed as mean \pm standard deviation. Significant P-values $(<0.05)$ are presented in italic. 
years), BMI and nutritional status (as shown in Table 1). Statin users were physically more active and had a higher socioeconomic status.

\section{Sarcopenia}

Table 2 presents comparison of functional capacity between statin users and controls. Maximal voluntary quadriceps muscle torque was higher in statin users $(25.3 \pm 7.5 \mathrm{~kg})$ than in controls $(20.5 \pm 6.3 \mathrm{~kg}, \mathrm{P}<0.001)$. Statin users performed also better at timed up and go $(9.7 \pm 2.1 \mathrm{~s}$ in statin users vs. $10.8 \pm 2.6 \mathrm{~s}$ in controls, $\mathrm{P}=0.01)$. Maximal handgrip strength did not differ between the two groups $(20.6 \pm 4.3 \mathrm{~kg}$ in statin users vs. $19.9 \pm 3.8 \mathrm{~kg}$ in controls, $\mathrm{P}=0.47$ ). Total lean body mass, as well as leg and arm lean mass (data not shown) did not differ between the two groups.

Various confounding factors influenced muscle strength. Quadriceps absolute force was correlated with socioeconomic status $(\mathrm{r}=0.59, \mathrm{P}<0.0001)$, timed up and go $(\mathrm{r}=-0.46$, $\mathrm{P}<0.01)$, physical activity $(\mathrm{r}=0.32, \mathrm{P}<0.001)$, muscle pain $(\mathrm{r}=-0.25, \mathrm{P}=0.004)$, age $(\mathrm{r}=-0.18$, $\mathrm{P}=0.04$ ). BMI, drug use or lapse of statin use were not correlated to quadriceps absolute strength. When the same correlations were performed with quadriceps specific force, age did not correlate any more as shown in Table 3.

Table 4 expresses the influence of these factors on the relation of statin intake and quadriceps specific force. After adjusting for confounding factors (BMI, muscle pain, physical activity, timed up and go and socioeconomic status), the effect of statin use was not significant, while socioeconomic status determined much of the variation in quadriceps specific force. The influence of socioeconomic status on strength was big ( $\beta$ coefficient 0.63 ) and changed the coefficient of determination (R2) from 0.25 to 0.46 ( 0.44 excluding TUG). Therefore, socioeconomic status was a stronger predictor of quadriceps specific force than use of statins. Adding TUG and socioeconomic status in the complete model changed muscle pain and physical activity into non-significant factors regarding quadriceps specific force. This was expected, since leg muscle pain, TUG and quadriceps strength are all correlated with each other and physical activity was dependent of socioeconomic status $(\mathrm{r}=0.34, \mathrm{P}<0.001)$.

Hand specific force, timed up and go and lean body mass were only affected by BMI, but not by statins (data not shown).

Since socioeconomic status was a strong predictor of muscle strength, functional capacity measures and body composition were compared between women with a high vs. low socioeconomic status (see Table 5). Women belonging to the high socioeconomic group had better quadriceps specific force $(\mathrm{P}<0.001)$ timed up and go performance $(\mathrm{P}<0.001)$ and lower body fat mass $(\mathrm{P}<0.02)$ than the group of low socioeconomic status. Lean body mass and was similar in both groups.

\section{Muscle pain and creatine kinase}

Muscle pain was reported in $42 \%$ of the statins users and in $35 \%$ of the control subjects $(\mathrm{P}=0.41)$. Serum creatine kinase (CK) levels did not differ between statin users and controls and were under the normal upper limit of 192 IU/L for women $(104 \pm 44$ in statin users vs. $92 \pm 40 \mathrm{IU} / \mathrm{L}$ in controls, $\mathrm{P}=0.13)$.

\section{Discussion and Conclusions}

The results of this study suggest that low dose $(<20 \mathrm{mg})$ statin treatment does not increase sarcopenia in elderly women, since we did not demonstrate deterioration in functional capacity or in lean body mass. To our best knowledge, this is the first study that investigated the effects of low-dosage statin treatment on sarcopenia in elderly women, measuring functional capacity by isokinetic quadriceps-, isometric hand grip strength as well as timed up and go and lean body mass.

The unexpected finding that statin users had better functional capacity (with regard to quadriceps muscle strength and timed up and go) was largely explained by the difference in socioeconomic status between the study groups. Subjects with a higher socioeconomic status were physically more active but did not have more lean body mass. Since only amount of lean body mass was measured by DEXA in our study, nothing can be said about differences in muscle quality between the two groups. It is possible that muscle quality is affected by socioeconomic status due to better nutritional quality. However, handgrip strength, a widely used indicator of nutritional quality in the elderly, 17 did not differ between socioeconomic levels. Since handgrip strength is a static measure that cannot be trained, the difference in physical activity is probably an important factor distinguishing the two groups in our study population. Improved muscle quality in the higher socioeconomic classes can be explained by better access to and higher quality of health care. Furthermore, differences in amount and type of physical and social activities can improve physical status. Another factor that could influence muscle quality is mus-

Table 3. Confounding factors related to quadriceps specific force $(\mathrm{kg} / \mathrm{kg})^{*}$

\begin{tabular}{lc} 
Variable & $\begin{array}{c}\text { Quadriceps } \\
\text { specific } \\
\text { force } \\
(\mathrm{kg} / \mathrm{kg})\end{array}$ \\
Socioeconomic status & $0.62(<0.001)$ \\
Timed up and go (sec) & $-0.44(<0.01)$ \\
\hline Physical activity & $0.34(<0.001)$ \\
Muscle pain & $-0.30(<0.001)$ \\
\hline BMI $\left(\mathrm{kg} / \mathrm{m}^{2}\right)$ & $-0.21(0.02)$ \\
Age (y) & $-0.10(0.18)$ \\
\hline Drug use & $0.02(0.73)$ \\
\hline
\end{tabular}

*Values expressed as spearman correlation coefficient (p-value). Significant P-values $(<0.05)$ are presented in italic.

Table 4. Effect of statins on quadriceps specific force $(\mathrm{kg} / \mathrm{kg})$ after controlling for confounding factors.*

\begin{tabular}{lccc} 
Variable & Model 1 & Model 2 & Model 3 \\
Statins & 0.35 & 0.33 & 0.15 \\
BMI $\left(\mathrm{kg} / \mathrm{m}^{2}\right)$ & -0.03 & -0.02 & 0.01 \\
\hline Muscle pain & - & -0.33 & -0.19 \\
Physical activity & - & 0.32 & 0.10 \\
\hline TUG $(\mathrm{sec})$ & - & - & -0.07 \\
Socioeconomic status & - & - & 0.63 \\
\hline Coefficient of determination $\left(\mathrm{R}^{2}\right)$ & 0.14 & 0.25 & 0.46
\end{tabular}

*Model 1. Quadriceps specific force controlled for effects of statins and BMI. Model 2. Added influence of physical activity and muscle pain. Model 3. Included also factor timed up and go and socioeconomic status. Data are presented as $\beta$ coefficients of multiple linear regression unless stated otherwise. Significant P-values $(<0.05)$ are presented in italic.

Table 5. Functional capacity comparing women from high versus low socioeconomic status.*

\begin{tabular}{lccc} 
Variable & High $(\mathrm{N}=72)$ & Low $(\mathrm{N}=56)$ & $\mathrm{P}$ \\
Quadriceps specific force $(\mathrm{kg} / \mathrm{kg})$ & $2.5 \pm 0.5$ & $1.7 \pm 0.5$ & $<0.001$ \\
Hand specific force $(\mathrm{kg} / \mathrm{kg})$ & $5.9 \pm 1.3$ & $5.8 \pm 1.1$ & 0.40 \\
\hline Timed up and go $(\mathrm{sec})$ & $9.5 \pm 1.9$ & $11.1 \pm 2.6$ & $<0.001$ \\
Total lean body mass $(\mathrm{kg})$ & $35.8 \pm 4.1$ & $36.4 \pm 4.6$ & 0.42 \\
\hline Total fat mass $(\mathrm{kg})$ & $24.0 \pm 6.7$ & $27.3 \pm 8.6$ & 0.02 \\
\hline
\end{tabular}

*Values expressed as mean \pm standard deviation. Significant P-values $(<0.05)$ are presented in italic. 
cle fat infiltration. Myosteatosis is associated with reduced isokinetic leg strength in older adults, ${ }^{18}$ probably due to increased muscle inflammation. ${ }^{19}$ Physical activity can reduce muscle fat infiltration, improving muscle function without increasing muscle mass. ${ }^{18}$ Our results are in accord with these findings, because subjects with higher socioeconomic status performed more physical activities, had lower fat mass and better muscular function, although no imaging techniques measuring muscle fat infiltration were used. Comparing muscle strength and timed up and go stratifying for socioeconomic status and physical activity, revealed no differences between statin users and controls, indicating that statin use did not affect functional capacity in our population. Finally, since only dynamic muscle strength measures differed between statin users and controls, statins at low dose, may improve vascular performance by influencing aerobic mechanisms.

Statin dose and age are important factors regarding the risk of developing myopathy. 9,20 In our study, low-dose ( $\leq 20 \mathrm{mg}$ ) statin intake was not associated with deteriorated functional capacity in elderly women. Our results on timed up and go and handgrip strength are in line with two other observational studies.21,22 One longitudinal study reported deteriorated quadriceps muscle function due to statin use. ${ }^{23}$ However, younger subjects were included (mean age 62 years) and a static measure of leg muscle strength was used. Unfortunately, the authors did not discuss if these effects were related to statin dose. Reduced strength was not reported in a randomized controlled trial using 12 week $80 \mathrm{mg}$ simvastatin treatment in 10 subjects (55-76 years). ${ }^{24}$ On the contrary, superior leg functioning was even found in statin users, including 392 elderly men with and without peripheral artery disease, as measured by $4 \mathrm{~m}$ walking performance. ${ }^{25}$ However, this study population consisted of only men with a mean age of 70 years and again, statin dose was not reported. Finally, a recent review regarding six studies related to statins and muscle strength reported insufficient data to determine if statins reduce muscle strength. ${ }^{26}$ In general, since dose and types of statins were not always reported, it is difficult to compare results. Therefore, future studies are needed assessing the effect of different doses and types of statin intake and muscle strength.

Age is an important factor relating to sarcopenia. Nevertheless is not surprising that age was not related to quadriceps specific force, although it was related to absolute quadriceps strength. Since lean mass was also correlated to age $(\mathrm{r}=-0.18, \mathrm{P}=0.039)$, the specific force (absolute torque/lean mass) did not correlate anymore.

Muscle pain was reported in both statin users as controls. Therefore, it is expected that creatine kinase levels (as a measure of muscle damage) did not differ between the groups. Apparently, low dose statin use does not elicit myopathy. 27 Thus, future similar studies should also include participants taking higher statin doses to compare the effects on functional capacity with users of low dose statins.

Our study has a number of limitations. Since a cross-sectional design was used, statin effects could not be measured within persons and various between-person factors could have influenced our results. Compliance to statin therapy was not tested, and low rates of compliance to statin therapy in older adults are reported. ${ }^{28,29}$ Furthermore, assessment of muscle pain, statin treatment duration and physical activity relied on self-report. Future studies should include within-person comparisons of functional capacity with clear assessment of statin dose and duration.

In conclusion, in this study sarcopenia in elderly women was influenced by socioeconomic status but not by statin use.

\section{References}

1. Mann D, Reynolds K, Smith D, Muntner P. Trends in statin use and low-density lipoprotein cholesterol levels among US adults: impact of the 2001 National Cholesterol Education Program guidelines, Ann Pharmacotherapy 2008; 42 : 1208-15.

2 Bruckert E, Hayem G, Dejager S, Yau C, Bégaud B. Mild to moderate muscular symptoms with high-dosage statin therapy in hyperlipidemic patients. The PRIMO Study. Cardiovascular Drugs and Therapy 2005;19:403-14.

3. Phillips PS, Haas RH, Bannykh S, et al. Statin-Associated Myopathy with Normal Creatine Kinase Levels. Ann Intern Med 2002;137:581-5.

4. Omar MA, Wilson JP. FDA adverse event reports on statin-associated rhabdomyolysis. The Annals of Pharmacotherapy 2002;36:288-95.

5. Dirks AJ, Jones KM. Statin-induced apoptosis and skeletal myopathy. Am J Physiol Cell Physiol 2006;291:1208-12.

6. Golomb BA, Evans MA. Statin adverse effects: a review of the literature and evidence for a mitochondrial mechanism. Am J Cardiovasc Drugs 2008;8:373-418.

7. Kiortsis DN, Filippatos TD, Mikhailidis DP, et al. Statin-associated adverse effects beyond muscle and liver toxicity. Atherosclerosis 2007;195:7-16.

8. Newman AB, Kupelian V, Visser M, et al. Strength, but not muscle mass, is associated with mortality in the health, aging and body composition study cohort. J Gerontol A Biol Sci Med Sci 2006;61:72-77.

9. Hansen KE, Hildebrand JP, Ferguson EE, Stein JH. Outcomes in 45 Patients With Statin-Associated Myopathy. Arch Intern Med 2005;165:2671-6.

10. Folstein MF, Folstein SE, McHugh PR. Mini-mental state. A practical method for grading the cognitive state of patients for the clinician. J Psychiatr Res 1975;12:18998.

11. Bunout D, Barrera G, de la Maza MP, et al. Effects of enalapril or nifedipine on muscle strength or functional capacity in elderly subjects. A double blind trial. J Renin Angiotensin Aldosterone Syst 2009;10:7784.

12 Guigoz Y, Vallas BJ, Garry PJ. Min Nutritional assessment: A practical assessment tool for grading the nutritional state of elderly patients. Facts Res Gerontol 1994;4:15-59.

13. Bunout D, Barrera G, Leiva L, et al. Effects of vitamin D supplementation and exercise training on physical performance in Chilean vitamine D deficient elderly subjects. Exp Gerontol.2006; 41:746-752.

14. Deones VL, Wiley SC, Worrell, T. Assessment of quadriceps muscle performance by a hand-held dynamometer and an isokinetic dynamometer. JOSPT 1994;20:296-301.

15. Callaghan John J. The adult knee. Ann Rheum Dis. 2003;62:1047-8.

16. Rockwood K, Awalt E, Carver D, MacKnight C. Feasibility and measurement properties of the functional reach and the timed up and go tests in the Canadian study of health and aging.. J Gerontol A Biol Sci Med Sci 2000;55:70-3.

17. Norman K, Stobäus N, Gonzalez MC, et al. Hand grip strength: Outcome predictor and marker of nutritional status. Clin Nutr. 2011;30:135-42.

18. Goodpaster BH, Chomentowski P, Ward BK, et al. Effects of physical activity on strength and skeletal muscle fat infiltration in older adults: a randomized controlled trial. J Appl Physiol 2008;105: 14981-503.

19. Nawrocki AR, Scherer PE. The delicate balance between fat and muscle: adipokines in metabolic disease and musculoskeletal inflammation. Current Opinion in Pharmacology 2004;4:281-9.

20. Harper C, Jacobsen T. The broad spectrum of statin myopathy: from myalgia to rhabdomyolisis. Curr Opin Lipidol 2007;18: 401-8.

21. Agostini JV, Tinetti ME, Han L, et al. Effects of Statin Use on Muscle Strength, Cognition, and Depressive Symptoms in Older Adults. J Am Geriatr Soc 2007;55: 420-5. 
22. Ashfield TA, Syddall HE, Martin HJ, Dennison EM, Cooper C, Aihie Sayer A. Grip strength and cardiovascular drug use in older people: findings from the Hertfordshire Cohort Study. Age Ageing 2010;39:185-91.

23. Scott D, Blizzard L, Fell J, Jones, G. Statin therapy, muscle function and falls risk in community-dwelling older adults. QJ Med 2009;102:625-33.

24. Traustadóttir T, Stock A, Harman M. High- dose statin use does not impair aerobic capacity or skeletal muscle function in older adults. AGE 2008;30:283-91.

25. McDermott MM, Guralnik JM, Greenland P, et al. Statin use and leg functioning in patients with and without lower-extremity peripheral arterial disease. Circulation 2003;107:757-61.

26. Krishnan GM, Thompson PD The effects of statins on skeletal muscle strength and excercie performance. Current Opinion in
Lipidology 2010;21:324-8.

27. Phillips PS, Haas RH, Bannykh S, et al. Statin-associated myopathy with normal creatine kinase levels. Ann Intern Med 2002;137:581-5.

28. Minhas, R. Statins in primary care: bridging the treatment gap: persistence in practice. Br J Cardiology 2004;11:487-91.

29. Howell N, Trotter R, Mottram DR, Rowe D. Compliance with statins in primary care. Pharm J 2004;272:23-6. 